\title{
Results from Geometric and Radiometric Calibration of TerraSAR-X
}

\author{
B. Bräutigam, M. Schwerdt, M. Bachmann, B. Döring \\ German Aerospace Center (DLR), Microwaves and Radar Institute \\ Oberpfaffenhofen, D-82234 Wessling, Germany \\ Phone: 0049-8153-283121, Fax: 0049-8153-281449 \\ Benjamin.Braeutigam@dlr.de
}

\begin{abstract}
TerraSAR-X is a scientific and commercial SAR mission to be launched in June 2007. As it provides highresolution products for a multitude of operation modes and incidence angles, the radiometric and geometric calibration of TerraSAR-X has to ensure the product quality and the correct in-orbit operation of the entire SAR system.

This paper describes the calibration activities for TerraSAR-X and the dedicated tasks to be performed during the five months commissioning phase. Results from on-ground tests are discussed with respect to geometric and radiometric calibration of the TerraSAR-X system.
\end{abstract}

\section{INTRODUCTION}

The first German SAR satellite for commercial and scientific applications, TerraSAR-X, will be launched in June 2007 [1]. TerraSAR-X will fly in a sun-synchronous orbit at an altitude of $514 \mathrm{~km}$. Its mission duration is designed for five years. The SAR instrument is designed to cover a wide range of swath positions and to operate in different operation modes by switching the instrument over a multitude of over 10,000 antenna beams. For the various antenna beams, an active phased array antenna electronically forms antenna radiation patterns. The array consists of 384 radiating sub-arrays for horizontal and vertical polarisation arranged in a matrix of 12 panels with 32 rows. Active transmit/receive (T/R) modules individually adjust the array elements in gain and phase for shaping and steering the antenna pattern in azimuth and elevation direction. Important system parameters are listed in Table I.

The success or failure of the mission essentially depends on the calibration of the TerraSAR-X system ensuring the product quality and the correct in-orbit operation of the entire SAR system. The essential task of calibrating TerraSAR-X is to estimate and correct systematic error contributions throughout the complete SAR system and to tie-down image information (magnitude and phase) to reference units in geophysical terms. The quality of this calibration process depends on the inherent stability of the radar system and the capability to determine and monitor the radiometric and geometric characteristics [2]-[4].

Due to the high degree of flexibility of TerraSAR-X and a tight performance with an absolute radiometric accuracy better than $1 \mathrm{~dB}$, it becomes clear that a conventional calibration approach is not feasible, i.e. the real measurement of all antenna beams in all operation modes, as performed for the ASAR instrument of ENVISAT across the rainforest [5]. Hence, a new, more efficient, and affordable calibration concept has been developed [3],[4]. The key element of this calibration concept is a novel antenna model approach [4],[6]. The TerraSAR-X antenna model is utilised for generating all reference antenna patterns and for beam optimisation.

The paper describes the in-orbit calibration procedure and the different activities performed before and after launch of TerraSAR-X. It shows the calibration status until satellite launch and gives an overview of the planned activities during the five months commissioning phase.

TABLE I

SYSTEM PARAMETERS OF TERRASAR-X

\begin{tabular}{ll}
\hline Center Frequency & $9.65 \mathrm{GHz}$ \\
\hline Bandwidth & $300 \mathrm{MHz}$ max \\
Antenna Array & 12 panels by 32 rows \\
& (384 T/R Modules) \\
Antenna Size & $4.8 \mathrm{~m}$ in azimuth, \\
& $0.8 \mathrm{~m}$ in elevation \\
Polarisation & H and V \\
& (single, dual, quad) \\
Look Angle Range & $15^{\circ}-60^{\circ}$ \\
Operation Mode & StripMap, ScanSAR, Spotlight \\
\hline
\end{tabular}

\section{SCOPE OF TERRASAR-X CALIBRATION}

Performed during commissioning of a SAR system, the objective of in-orbit calibration can be sub-divided into three major tasks:

- Geometric Calibration: to assign the SAR system to the geographic location on the earth surface.

- Relative Radiometric Calibration: for radiometric correction of SAR data within an illuminated scene.

- Absolute Radiometric Calibration: the measurement of the SAR system against standard targets with well known geophysical characteristics.

The calibration steps rely on the antenna model which was validated on-ground. In addition to the tasks described above, the verification of this antenna model has to be executed in orbit. This ensures the provision of the antenna patterns of all beams and the gain offset between adjoining beams. 


\section{STRATEGY}

In addition to the complexity of TerraSAR-X and the corresponding challenges described before, the main goal after launch of the satellite is to provide calibrated and verified SAR data products as far as possible at the latest by the end of the commissioning phase. Thus, a strategy for an efficient but robust calibration approach has been developed. The successive baseline calibration processes are:

1. Geometric Calibration

2. Antenna Pointing Determination

3. Antenna Model Verification

4. Relative Radiometric Calibration

5. Absolute Radiometric Calibration

Furthermore, by applying the novel T/R Module Characterisation Method [7],[8], the actual antenna settings will be derived in orbit. Individual and simultaneous monitoring of all $\mathrm{T} / \mathrm{R}$ modules is possible in-flight under the most realistic instrument conditions. This saves valuable time during commissioning and in case of antenna module failure or degradation. In the following the process steps of the inorbit calibration are described in detail.

\section{A. Geometric Calibration}

The purpose of the geometric calibration is the geometric assignment of the SAR system to the earth's surface. Two effects can influence the correct localisation of the product:

- $\quad$ systematic azimuth shifts and

- the internal electronic delay of the instrument.

For both effects, corner reflectors are deployed as they have no additional electronic delay and consequently no additional source of error occurs.

\section{B. Antenna Pointing Determination}

An important task is the determination of beam pointing errors coming from mechanical and electrical antenna mispointing as well as from attitude control offsets. These errors are measured in elevation and in azimuth using an appropriate notch pattern over rain forest and by ground receivers. But also a Doppler analysis is performed to evaluate the error in flight direction.

\section{Antenna Model Verification}

The characterisation of the antenna is based on a precise antenna pattern model, which has to be validated against precisely measured near field patterns on-ground before launch and verified in-orbit by in-flight measurements over homogeneous targets (rain forest) and deployed ground receivers. One example of the validation on-ground performed by Astrium is shown in Fig. 1. The solid blue curve is the measured elevation pattern of one panel and the red curve of that derived by the model. The deviation of the shape within the main lobe is less than $0.15 \mathrm{~dB}$. The results of the validation of the antenna model are described in [6].

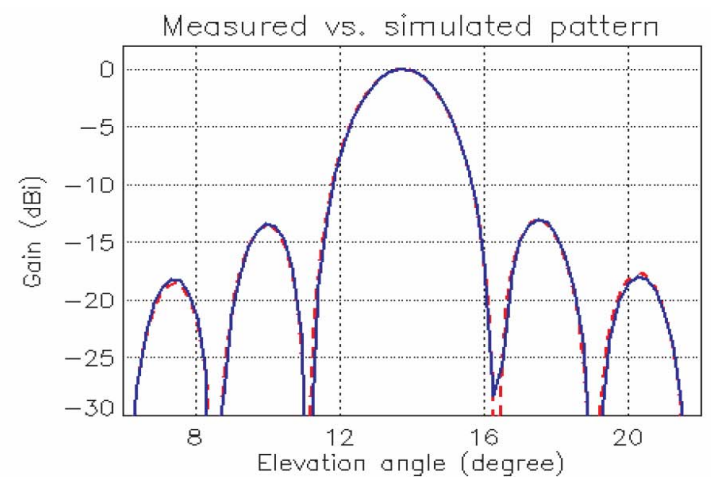

A)

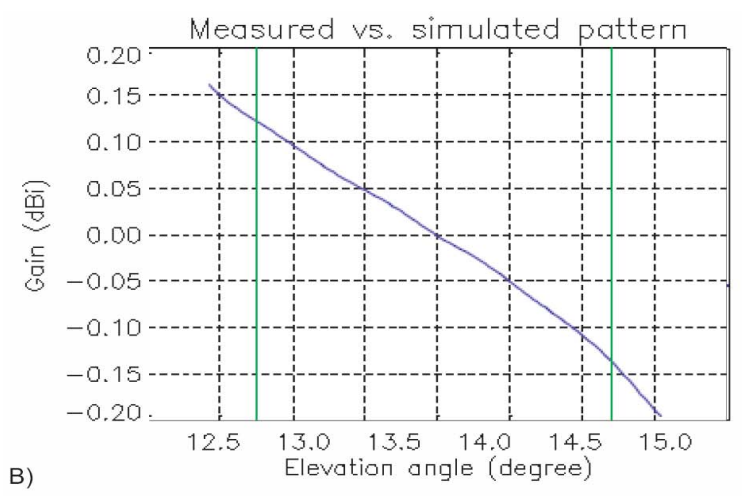

Fig. 1 A) comparison between an exemplary beam measured on ground (blue/solid) and derived by the antenna model (red/dotted), B) difference within the main beam between model and measurement.

The in-orbit verification will be performed by three selected beams with low, medium and high incidence angles. For this, the elevation patterns are measured over rain forest and compared to the pre-calculated patterns. Furthermore, the transmitted pulses are recorded on-ground by deployed ground receivers. With this method the transmit pattern of the TerraSAR-X antenna can be really measured in-flight verifying the one-way azimuth patterns.

One example of really measured antenna patterns of ASAR/ENVISAT is shown in Fig. 2. The reduced amplitudes indicate the switching of the instrument from beam to beam and the envelopes represent the one-way azimuth pattern of the corresponding beam.

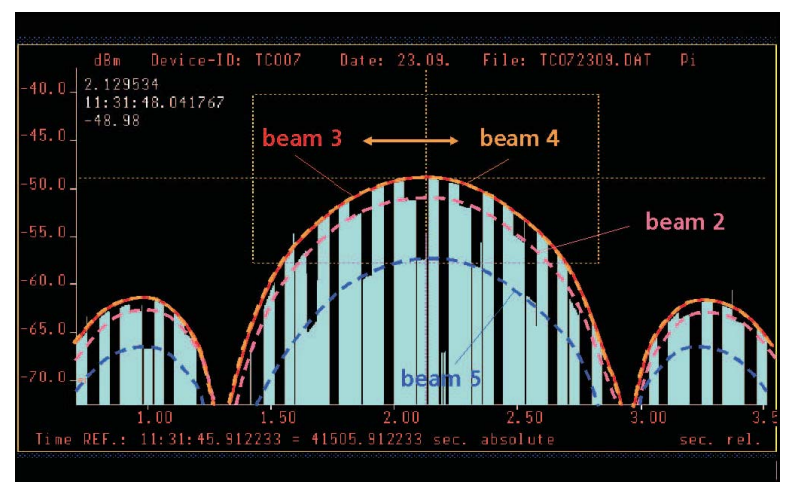

Fig. 2 One-way (transmit) azimuth antenna patterns of the ASAR/ENVISAT instrument in ScanSAR operation measured by a ground receiver. 
The relative gain variation from beam to beam will be characterised over homogeneous targets in ScanSAR operation. Thus, the effort of the absolute radiometric calibration can be reduced down to a few beams instead of measuring TerraSAR-X against deployed calibration targets for all operation modes.

The antenna model provides a software tool that accurately determines all beam patterns based on detailed characterisation of the radar antenna and knowledge of the antenna control parameters. Beyond these capabilities the antenna pattern module also features a tool for generating optimised beam coefficients under given constraints. The major advantage of this beam optimisation is the dynamic recalibration in the event of T/R Module degradations [8] during the mission.

\section{Relative and Absolute Radiometric Calibration}

After in-orbit verification of the antenna model the beam patterns required for radiometric correction of SAR data within an illuminated scene are calculated by the antenna model for all operation modes and all incidence angles.

In order to reduce calibration effort, the absolute radiometric calibration is likewise based on the antenna model, i.e. the real measurements over point targets with known radar cross section are performed on a reduced set of beams. The absolute calibration factors of all other beams and modes are derived via the antenna model.

Hence, by applying the antenna model, the effort for both the relative and the absolute radiometric calibration as well as the duration of the calibration campaigns can be significantly reduced to keep the commissioning phase of five months.

\section{CALIBRATION CAMPAIGNS AFTER TERRASAR-X LAUNCH}

External calibration is based on SAR data acquisition over test sites with well-known backscatter characteristics. Basically, these sites can be homogenous areas like the Amazonian rain forest or scenes with point targets like corner reflectors or active transponders.

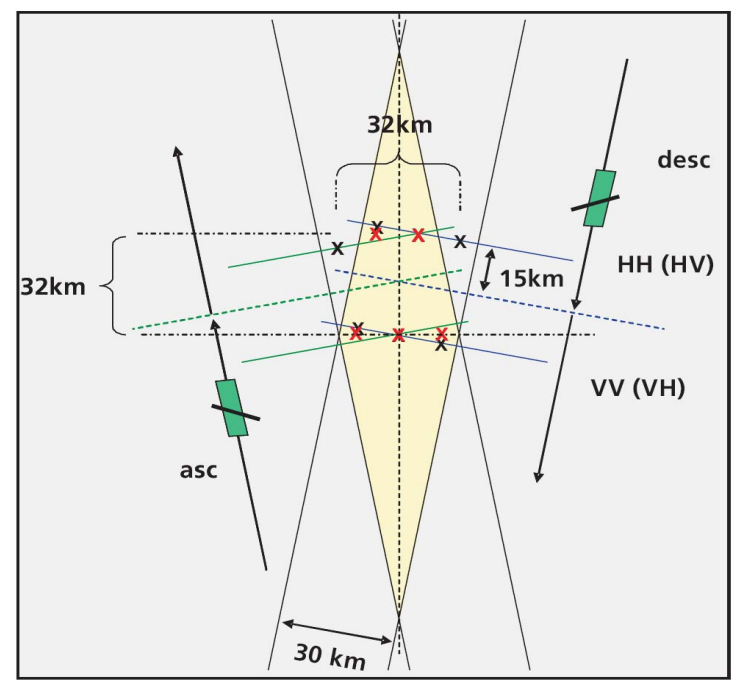

Fig. 3 Test site configuration for TerraSAR-X. The test site consists of 7 target positions and encloses an area of $32 \mathrm{~km} \times 32 \mathrm{~km}$.
To account for the restricted time of calibration campaigns the number of passes and places of test sites is optimised versus cost and time effort by calibrating several beams and polarisation modes with the same test site.

\section{A. Coverage}

As single beams do not cover the complete globe, the selection of test sites is constrained to the availability of respective beams on ground. The coverage on the earth's surface needs to be evaluated with respect to the required number of point target and rain forest measurements assumed in the radiometric accuracy budget. In order to obtain as many overflights as possible over calibration targets, special test site configurations have been selected in the crossing points of ascending and descending passes.

\section{B. Test Site Configuration}

Driven by the radiometric accuracy budget within a StripMap swath of $30 \mathrm{~km}$ range two targets are deployed in near range, at swath centre and in far range, respectively, see Fig 3. The targets must be sufficiently separated to avoid ambiguities in the image. Due to a slight displacement, most of the positions (red crosses) can be applied twice for descending and ascending orbit. Thus the number of required target positions within a test site could be reduced extremely.

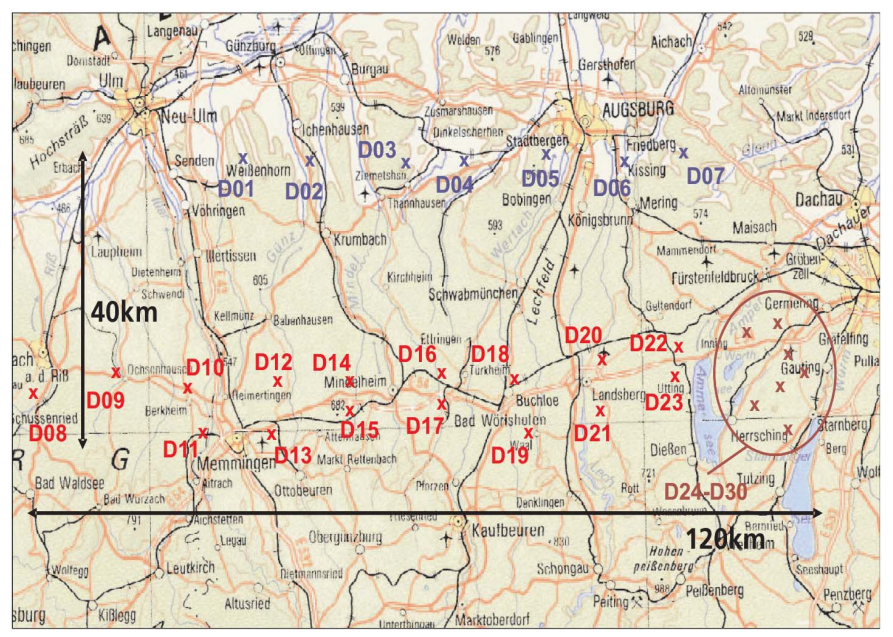

Fig. 4 DLR calibration field in South Germany consisting of 30 target positions and enclosing an area of $120 \mathrm{~km} \times 40 \mathrm{~km}$.

\section{DLR Calibration Field}

To achieve a high number of passes during a restricted time of commissioning TerraSAR-X a calibration field has been established enclosing an area of $120 \mathrm{~km} \mathrm{x} 40 \mathrm{~km}$ and consisting of 30 target positions, see Fig 4. By this calibration field several crossing over areas of the same and of different beams are covered. Considering the test site configuration described above a re-use of most of the target positions for several passes (e.g. ascending and descending) is possible.

Mainly for logistic reasons this calibration field was selected in South Germany which can be operated and maintained from DLR Oberpfaffenhofen, see Fig 4. 


\section{DLR Calibration Facility}

The realisation of the TerraSAR-X calibration concept and the implementation of the procedures described above requires a calibration facility that is well-equipped with ground calibration hardware as well as software tools for evaluating the measurements. For this purpose the Microwave and Radar Institute has developed and established the following reliable and accurate ground equipment:

- accurate ground targets like corner reflectors and transponders precisely surveyed for geometric and radiometric calibration,

- ground receivers measuring the one-way azimuth pattern of the SAR antenna during an overflight,

- different analysis and evaluation tools, like the mentioned antenna model providing an estimation of the actual antenna pattern, or CALIX, a software tool for point target analysis, featuring measurements of impulse response function parameters and the absolute calibration factor. Furthermore a geometric analysis for accurately surveyed targets can be performed yielding the internal delay of the instrument and the datation accuracy. Fig. 5 shows a screen-shot of this tool.

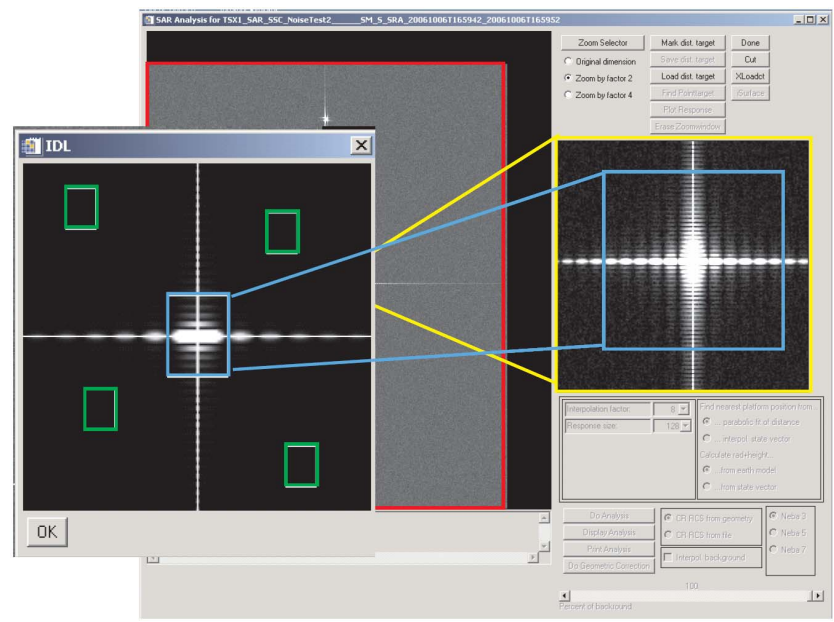

Fig. 5 Screenshot of CALIX, a software for point target and geometric analysis of SAR products.

The existing infrastructure is well prepared for executing calibration campaigns over large test sites. Accordingly, reliable and accurate ground equipment is guaranteed during the whole mission life time.

\section{SUMMARY}

In preparation of the TerraSAR-X satellite launch in June 2007 DLR has thoroughly planned the calibration procedures to be performed during the five months commissioning phase. By applying the antenna model as key element of the in-orbit calibration strategy, most of the antenna characterisation effort has been shifted from in-flight to on-ground activities. An efficient but accurate way to accomplish a short commissioning phase has been developed as only a few beams are measured in orbit.

A multitude of powerful software tools has been established within on-ground tests to support the evaluation of calibration measurements. Therefore, corresponding test site configurations have been developed and arranged in South Germany, supported by precise and sophisticated ground equipment from the DLR Oberpfaffenhofen calibration facility.

After satellite launch calibration activities consume most of the time during the five months commissioning phase. The first measurements begin three weeks after launch. Over 400 acquisitions across the Amazonian rain forest are planned. The various calibration measurements in Germany require ground support by professional calibration teams performing more than 60 campaigns in a time frame of less than five months. This amount of measurements is sufficient for calibrating over 10,000 antenna beams.

The results from the in-orbit calibration are a pre-requisite for successful SAR product verification as they define the geometric and radiometric performance of TerraSAR-X.

\section{REFERENCES}

[1] R. Werninghaus, "The TerraSAR-X Mission," in 6th European Conference on Synthetic Aperture Radar, Dresden, Germany, 2006.

[2] M. Schwerdt, B. Bräutigam, M. Bachmann, T. Molkenthin, D. Hounam, and M. Zink, "The Calibration of TerraSAR-X," in 6th European Conference on Synthetic Aperture Radar, Dresden, Germany, 2006.

[3] M. Schwerdt and D. Hounam, "Quality Control and Calibration of Future SAR Systems," in ODAS 2001, 3rd ONERA-DLR Aerospace Symposium, Paris, France, 2001.

[4] M. Schwerdt, D. Hounam, B. Bräutigam, and J. L. Alvarez-Pérez, "TerraSAR-X: Calibration Concept of a Multiple Mode High Resolution SAR," in IEEE: 25th International Geoscience And Remote Sensing Symposium, Seoul, Korea, 2005.

[5] M. Zink and B. Rosich, "Antenna Elevation Pattern Estimation from Rain Forest Acquisitions," in ENVISAT/ASAR Calibration Review (ECR) of ESTEC, European Space Agency (ESA), Noordwijk, Netherland, 2002.

[6] M. Bachmann, M. Schwerdt, B. Bräutigam, B. Grafmüller, A. Herschlein, and J. L. Alvarez-Pérez, "The TerraSAR-X Antenna Model Approach," in 2nd International ITG Conference on Antennas, Munich, Germany, 2007.

[7] D. Hounam, M. Schwerdt, and M. Zink, "Active Antenna Module Characterisation by Pseudo-Noise Gating," in 25th ESA Antenna Workshop on Satellite Antenna Technology, Noordwijk, Netherlands, 2002.

[8] B. Bräutigam, M. Schwerdt, M. Bachmann, and M. Stangl, "Individual T/R Module Characterisation of the TerraSAR-X Active Phased Array Antenna by Calibration Pulse Sequences with Orthogonal Codes," in 26th International Geoscience And Remote Sensing Symposium, Barcelona, Spain, 2007.

[9] J. L. Alvarez-Pérez, M. Schwerdt, D. Hounam, R. Torres, C. Buck, and M. Zink, "Antenna Pattern Optimization for the ENVISAT ASAR Antenna with Failed Elements," in 25th ESA Antenna Workshop on Satellite Antenna Technology, Noordwijk, Netherlands, 2002.

[10] B. Bräutigam, M. Schwerdt, and M. Bachmann, "The External Calibration of TerraSAR-X, a Multiple Mode SAR-System," in 6th European Conference on Synthetic Aperture Radar, Dresden, Germany, 2006. 